\title{
Clusters in dense matter and the equation of state
}

\section{S. Typel ${ }^{* a, b}$, G. Röpke ${ }^{c}$, T. Klähn ${ }^{d}$, D. Blaschke ${ }^{d}$, H. H. Wolter ${ }^{e}$ and M. Voskresenskaya ${ }^{b}$}

${ }^{a}$ Excellence Cluster Universe, Technische Universität München, Boltzmannstraße 2, 85748

Garching, Germany

${ }^{b}$ GSI Helmholtzzentrum für Schwerionenforschung GmbH, Planckstraße 1, 64291 Darmstadt, Germany

${ }^{c}$ Institut für Physik, Universtität Rostock, Universitätsplatz 3, 18055 Rostock, Germany

${ }^{d}$ Instytut Fizyki Teoretycznej, Uniwersytet Wrocławski, plac Maksa Borna 9, 50-204 Wrocław, Poland

${ }^{e}$ Fakultät für Physik, Universität München, Am Coulombwall 1, 85748 Garching, Germany

E-mail: S.typelegsi.de gerd.roepke@uni-rostock.de,

thomas.klaehnegooglemail.com david.blaschkelgmail.com,

hermann.wolter@physik.uni-muenchen.de, m.voskresenskaya@gsi.de

The equation of state of dense matter is an important ingredient in astrophysical models for supernovae and compact stars. The thermodynamical properties and the composition of matter below the nuclear saturation density is modified by the appearance of clusters and inhomogeneities. In this contribution a generalized relativistic mean-field model is presented that considers the formation and dissolution of light and heavy clusters in a microscopic approach. The model allows to describe dense matter consistently from very low densities where strong correlations are important up to and beyond nuclear saturation density where a quasi-particle description in a mean-field approach is very successful. Cluster formation affects the density dependence of the symmetry energy. This effect can be tested by a comparison of model calculations to experimental data obtained from the analysis of heavy-ion collisions. The aim of this work is to construct an improved equation of state in a range of densities, temperatures and neutron-proton asymmetries that is relevant for astrophysical applications.

11th Symposium on Nuclei in the Cosmos, NIC XI

July 19-23, 2010

Heidelberg, Germany

\footnotetext{
* Speaker.
} 


\section{Introduction}

When the era of nuclear fusion reactions ends, the last phases in the life of a star are reached. A massive star $\left(8 M_{\text {sun }} \lesssim M_{\text {star }} \lesssim 30 M_{\text {sun }}\right)$ will experience a core collapse producing a spectacular supernovae and will end as a neutron star or, for heavier masses, as a black hole [1]. The dynamical evolution of the core-collapse supernova and the static properties of the emerging neutron star are determined by the equation of state (EoS) of dense matter. It affects the energetics, chemical composition and transport properties of the matter under extreme conditions and sets the conditions for the synthesis of heavy elements in later stages of the evolution. Thus it is evident that the EoS is an essential ingredient in astrophysical models. Supernovae simulations cover a wide region of thermodynamical conditions. Typical values for the density $\rho$, the temperature $T$ and the electon fraction $Y_{e}$ lie in the ranges $10^{-9} \lesssim \rho / \rho_{\text {sat }} \lesssim 10$ (with the nuclear saturation density $\rho_{\text {sat }} \approx 2.5$. $\left.10^{14} \mathrm{~g} / \mathrm{cm}^{3}\right), 0 \mathrm{MeV} \leq k_{B} T \lesssim 25 \mathrm{MeV}\left(\hat{=} 2.9 \cdot 10^{11} \mathrm{~K}\right)$ and $0 \leq Y_{e} \lesssim 0.6$. Clearly, a global theoretical description of matter properties is required.

Many EoS have been developed in the past, from very simple parametrizations to sophisticated models. However, most of the investigations concentrated on detailed aspects, often restricted to particular condition, e.g. zero temperature or pure neutron matter. In many cases the results are not easily available in a form suitable for dynamical model calculations. Thus there are only few EoS actually used in simulations of core-collapse supernovae. The most well-known EoS are those by Lattimer and Swesty [2] and Shen et al. [3]. The most difficult problem is the description of the strongly interacting subsystem of hadronic matter and certain approximations are needed. In this contribution, we concentrate on a particular feature, the formation and dissolution of clusters in nuclear matter at densities below saturation. In previous EoS [2, 3] only nucleons, $\alpha$-particles and a representative heavy nucleus we considered. The suppression of the $\alpha$-particle was modeled with a phenomenological excluded-volume mechanism. Here, we consider a more microscopic approach that includes more light clusters and interactions between all constituents of the matter.

\section{Theoretical approaches}

The composition of nuclear matter depends strongly on density, temperature and neutronproton asymmetry and it affects the thermodynamical properties. There are essentially two different points of view in a theoretical description: 1. in a chemical picture, a mixture of different nuclear species and nucleons in chemical equilibrium is considered; typical approaches are nuclear statistical equilibrium (NSE) models and the virial or Beth-Uhlenbeck EoS; 2. in a physical picture, the emphasis is on the correlations caused by the strong interaction leading to the formation of clusters as bound states of nucleons; here quantum statistical (QS) or (Dirac) Brueckner-Hartree-Fock approaches are employed.

Both pictures face certain problems. In the former approach, the properties of the constituents are independent of the medium and the dissolution of nuclei at high densities has to be modeled in a phenomenological way. The interaction between the particles can be considered within the virial expansion that is valid, however, only at low densities. Extended NSE models with interactions have been discussed recently [ [ [ In the approaches of the latter picture the interaction between the nucleons has to be chosen carefully and the treatment of three-, four-, ... many-body correlations is 
a difficult task. The chemical picture is very successful at low densities, whereas at high densities around and above nuclear saturation usually nonrelativistic Hartree-Fock or relativistic mean-field (RMF) models are employed without explicit correlations. At densities just below nuclear saturation and not too high temperature the liquid-gas phase transition is observed with the formation of "pasta" phases where the matter is inhomogeneously distributed in space and the specific distributions depends strongly on surface effects and the long-range Coulomb interaction. It is the goal of this work to combine the two picture yielding a consistent interpolation between the low- and high-density limits.

The present model is discussed in detail in [5]. It combines a QS approach with a generalized RMF descripton of nuclear matter with clusters. The QS model is based on a finite-temperature Greens' function formalism where the nucleon number densities are expressed with help of spectral functions that depends on the particle's self-energy. The spectral function is expanded beyond the quasi-particle approximation and thus takes correlations into account. The result is a generalized Beth-Uhlenbeck description, similar to the virial EoS, with medium dependent binding energies of the occurring bound states and generalized phase shifts in the continuum states from the in-medium T matrix. For given temperature and nucleon densities, the corresponding chemical potentials and, by integration, the free energy density can be derived leading to a thermodynamically consistent EoS. Inside the medium, the nucleon properties are modfied as compared to free space with a self-energy shift of the quasiparticle energy and an effective mass. Similarly, the cluster properties change due to a shift of the quasi-particle energy from the nucleon self-energies and Pauli blocking. Effectively, the shifts of the cluster binding energies are density and temperature dependent, see, e.g., figure 1 in [5], They are calculated with an effective nucleon-nucleon potential.

The nucleon self-energies used in the QS approach are taken from a RMF model with densitydependent meson-nucleon couplings. The parameters of the model are fitted to properties of finite nuclei and the resulting nuclear matter parameters are very reasonable. The RMF model is extended to include deuterons, tritons, ${ }^{3} \mathrm{He}$ nuclei and $\alpha$-particles as additional degrees of freedom. Their medium-dependent binding energies are used in a parametrized form employing the results of the QS model. The field equations, as derived from the generalized RMF Lagrangian density, are solved selfconsistently in the mean-field approximation. The medium dependence of the couplings and the binding energies generates so-called rearrangement contributions in the self-energies and source densities of the meson fields that are essential for the thermodynamical consistency of the model.

\section{Clusters in dense matter and symmetry energy}

Presently, only the bound states of two-, three- and four-body correlations are considered in the extended RMF model with medium-dependent binding energy shifts. Within this approach, the Mott effect is observed, i.e. the light clusters dissolve with increasing medium density and the correct limits at low and high densities are found. See figures 3 and 4 in [5]. Deviations of thermodynamical properties from standard NSE results are already observed at densities as low as $10^{-3} \rho_{\text {sat }}$ where interaction and medium effects start to become important.

An application of the generalized RMF model with light clusters to the description of homogeneous nuclear matter predicts the occurrence of a liquid-gas phase transition in the phase dia- 

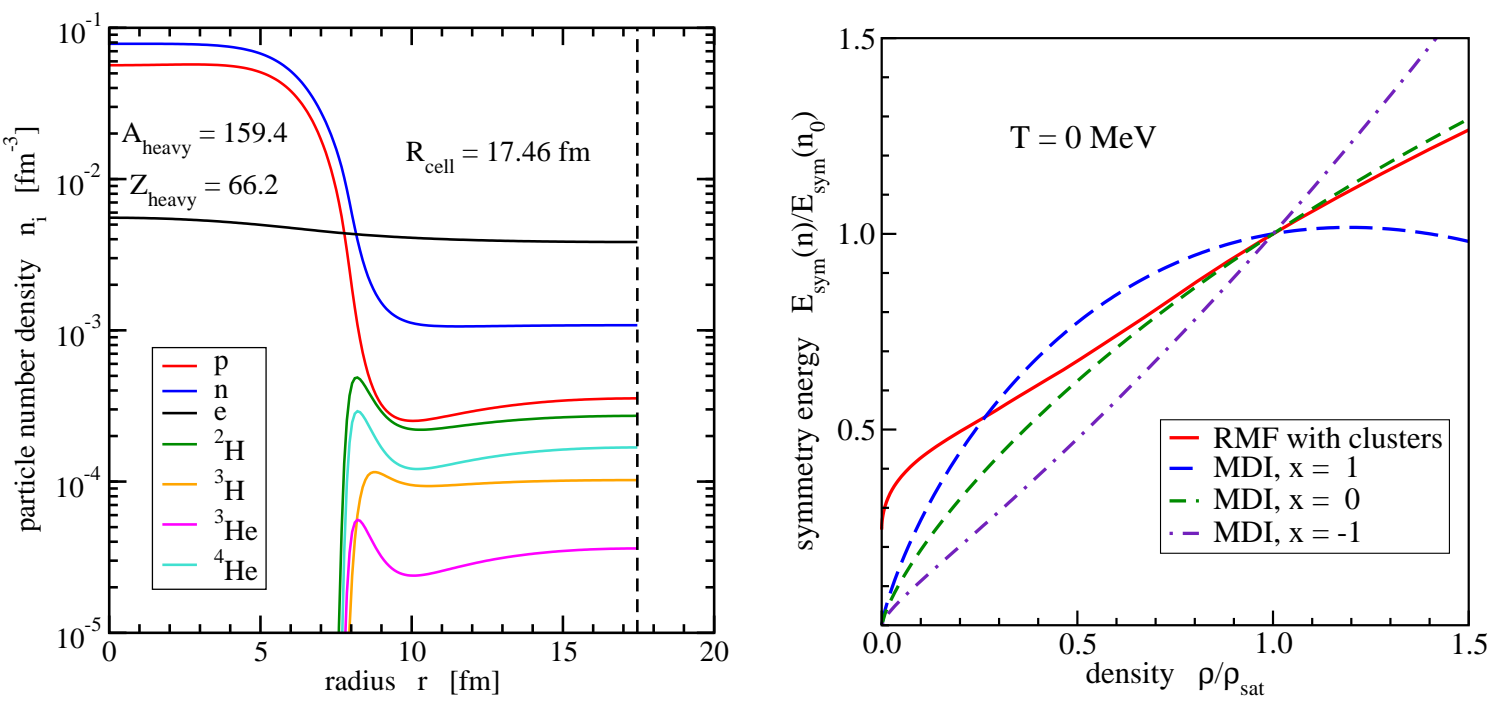

Figure 1: Left panel: Distribution of nucleons, light clusters and eletrons in a spherical Wigner-Seitz cell for temperature $T=5 \mathrm{MeV}$, baryon number density $n=0.01 \mathrm{fm}^{-3}$ and electron fraction $Y_{e}=0.4$. Right panel: Density dependence of the symmetry energy at $T=0 \mathrm{MeV}$ in the MDI model for various values of the slope parameter $x$ (dashed and dot-dashed lines) and in the generalized RMF model with clusters (red solid line).

gram with a mixed phase where macroscopic spatial regions of high and low density appear. This approach neglects surface and Coulomb effects that are crucial for the development of the pasta phases. A first step in the improvement of the model is a Wigner-Seitz cell calculation assuming spherical symmetry. It allows for the formation of a heavy nucleus that is surrounded by a gas of nucleons and light clusters. Electrons have to be included in the model for charge compensation. The left panel of figure 11 depicts preliminary results of the radial particle distribution inside the cell for particular conditions from a self-consistent calculation where all particles interact and the cell radius if found by a minimization of the free energy density.

The symmetry energy $E_{\text {sym }}$ of nuclear matter characterizes the dependence of the EoS on the neutron-proton asymmetry. Properties of neutron stars strongly depend on the density dependence of $E_{\mathrm{sym}}$ [6]. The value of the symmetry energy near nuclear saturation density is rather well known but the density dependence of $E_{\text {sym }}$ is intensely debated.

Traditional mean-field models of nuclear matter predict that the symmetry energy vanishes when the density approaches zero. An example is the symmetry energy in a model with a momentumdependent interaction (MDI) that was extensively in the simulation of HIC [7]. The parameter $x$ controls the slope of the symmetry energy and the extrapolation, see the right panel of figure 1 . However, the low-density behaviour is not correct. At low-densities in nearly neutron-proton symmetric systems, matter is inhomogeneous and clusters are formed yielding an increase of the binding energy. This is reflected by a larger symmetry energy in this density region with a finite value for $\rho \rightarrow 0$ at zero temperature. The generalized RMF model that takes the formation of clusters into account predicts the expected increase of the symmetry energy.

The symmetry energy at densities below saturation can be determined from heavy-ion collisions [8]. The analysis shows an enhanced symmetry energy at low densities and finite temperatures 
that is consistent with the EoS in the QS model calculation that includes cluster formation, see figure 1 in [8]. In constrast, a RMF calculation without clusters fails to describe the experimental data predicting much too low symmetry energies.

\section{Conclusions and outlook}

Correlations and the formation of clusters are important features in dense matter. They affects the equation of state relevant for astrophysical applications. The present generalized relativistic mean-field model in combination with a quantum statistical approach explicitly considers the appearance and dissolution of light and heavy clusters. It exhibits the correct limits at low and high densities and predicts an increase of the nuclear symmetry energy at low densities. A comparison with experimental data from heavy-ion collisions supports the theoretical model.

Presently, the parametrization of the density dependence of the couplings is revisited to include constraints from the virial EoS and nucleon-nucleon scattering [9]. Two-body correlations in the continuum will be included in the future. In addition, it is planned to consider more symmetries of the Wigner-Seitz cell beyond spherical to describe the transition from clustered matter at low densities to homogeneous matter at high densities. Finally, based on the new approach, an improved and better constrained EoS will be made available for astrophysical applications.

This research was supported by the DFG cluster of excellence "Origin and Structure of the Universe" and by CompStar, a Research Networking Programme of the European Science Foundation.

\section{References}

[1] M. Liebendörfer et al., Models and direct observables of core-collapse supernovae, in proceedings of 11th Symposium on Nuclei in the Cosmos, NIC XI, POS (NIC XI) 026.

[2] J. M. Lattimer and F. D. Swesty, A generalized equation of state for hot, dense matter, Nucl. Phys. A 535 (1991) 331.

[3] H. Shen el al., Relativistic Equation of State of Nuclear Matter for Supernova Explosion, Prog. Theor. Phys. 100 (1998) 1013.

[4] M. Hempel and J. Schaffner-Bielich, Statistical Model for a Complete Supernova Equation of State, arXiv:0911.4073.

[5] S. Typel et al., Composition and thermodynamics of nuclear matter with light clusters, Phys. Rev. C 81 (2010) 015803 [arXiv: 0908.2344 ].

[6] T. Klähn et al, Constraints on the high-density nuclear equation of state from the phenomenology of compact stars and heavy-ion collisions, Phys. Rev. C 74 (2006) 035802 [nucl-th/ 0602038 ].

[7] B. A. Li et al., Recent progress and new challenges in isospin physics with heavy-ion reactions, Phys. Rep. 464 (2008) 113.

[8] J. B. Natowitz et al., Symmetry Energy of Dilute Warm Nuclear Matter, Phys. Rev. Lett. 104 (2010) 202501 [arXiv:1001.1102].

[9] M. Voskresenskaya and S. Typel, Nuclear equation of state in the relativistic mean field model with density dependent coupling constants, in proceedings of 11th Symposium on Nuclei in the Cosmos, NIC XI, POS (NIC XI) 180. 